\title{
Ring-Opening of Epoxides with Amines for Synthesis of $\beta$-Amino Alcohols in a Continuous-Flow Biocatalysis System
}

\author{
Li-Hua Du ${ }^{1, *}$, Miao Xue ${ }^{1}$, Meng-Jie Yang ${ }^{1}$, Yue Pan ${ }^{1}$, Ling-Yan Zheng ${ }^{1}$, Zhi-Min Ou ${ }^{1}$ and \\ Xi-Ping Luo ${ }^{2, *}$ \\ 1 College of Pharmaceutical Science, Zhejiang University of Technology, Hangzhou 310014, China; \\ xuemiao@zjut.edu.cn (M.X.); yangmengjie@zjut.edu.cn (M.J.Y.); panyuezjut@163.com (Y.P.); \\ zhenglingyan1@163.com (L.-Y.Z.); oozzmm@zjut.edu.cn (Z.-M.O.) \\ 2 Zhejiang Provincial Key Laboratory of Chemical Utilization of Forestry Biomass, \\ Zhejiang A\&F University, Hangzhou 311300, China \\ * Correspondence: orgdlh@zjut.edu.cn (L.-H.D.); luoxiping@zafu.edu.cn (X.-P.L.); \\ Tel.: +86-18969069399 (X.-P.L.)
}

Received: 22 October 2020; Accepted: 27 November 2020; Published: 4 December 2020

\begin{abstract}
An efficient method for the preparation of $\beta$-amino alcohols catalyzed by lipase TL IM from Thermomyces lanuginosus in a continuous-flow reactor was developed. The eco-friendly biocatalyst combined with continuous-flow reaction technology displayed high efficiency in the synthesis of $\beta$-amino alcohols. The benign reaction conditions $\left(35^{\circ} \mathrm{C}\right)$ and short residence time $(20 \mathrm{~min})$, together with the use of low cost and readily available starting materials, make this synthetic approach a promising alternative to current $\beta$-amino alcohol synthesis.
\end{abstract}

Keywords: enzymatic synthesis; $\beta$-amino alcohols; reactor; epoxides; ring-opening reaction

\section{Introduction}

$\beta$-Amino alcohols are vital intermediates for the preparation of various bioactive compounds, chiral auxiliaries, unnatural amino acids and organocatalysts [1-6]. Moreover, $\beta$-amino alcohols play an increasingly key role in pharmaceuticals, where they are used as cardiovascular drugs (I), anti-asthma drugs (II), antimalarial drugs (III), antihypertensive drugs (IV), $\beta$-blockers, antibacterial and hypoglycemic drugs, etc. [7-10] (Figure 1). Given the significance of $\beta$-amino alcohols in organic synthesis and pharmaceutical development, the development of a flexible synthetic pathway to this moiety is desperately desirable. The asymmetric ring-opening (ARO) reaction of epoxides with amines is the most straightforward route to prepare $\beta$-amino alcohols. In recent years, several catalysts such as Lewis acids [11-13], metal-organic frameworks [14] and Bismuth (III) salts [15-18] have been reported for ring-opening reactions. However, these approaches usually require the use of costly reagents, high catalyst loadings, poor regioselectivity and also entail side effects, such as the rearrangement of epoxides to allyl alcohols under strong alkaline conditions or polymerization under strongly acidic conditions. Therefore, it is urgent to develop a simple, efficient and environmentally benign synthetic method.

Biocatalysis shows good selectivity in organic synthesis, hence making it an environmentally friendly alternative to chemical synthesis [19-22]. Lipases have been extensively explored in Michael reactions, esterification and Knoevenagel-Michael cascade reactions to synthesize a variety of vital molecules related to foods, pharmaceuticals, flavorings and fine chemicals [23-28]. However, so far the synthesis of $\beta$-amino alcohols from the ring-opening of epoxides with aromatic amines catalyzed by enzymes has rarely been studied. Lipase from Aspergillus oryzae has been reported to be used in the 
synthesis of $\beta$-amino alcohols through a one-pot reaction along with amines, epichlorohydrin and aromatic phenols. This reaction needs to be carried out under the synergistic action of phase transfer catalyst 1-butyl-3-methylimidazolium chloride salt ([BMIM]Cl) [29]. Pankaj [30] used Candida rugosa lipase to catalyze the ring-opening of epoxides with amines in diisopropyl ether (DIPE), and $70 \%-90 \%$ yields could be obtained in 8-12 h. Compared to chemical catalysis, enzymatic reactions are relatively mild, but they need extra auxiliary reagents or a longer reaction time to achieve the anticipated yield (Scheme 1).
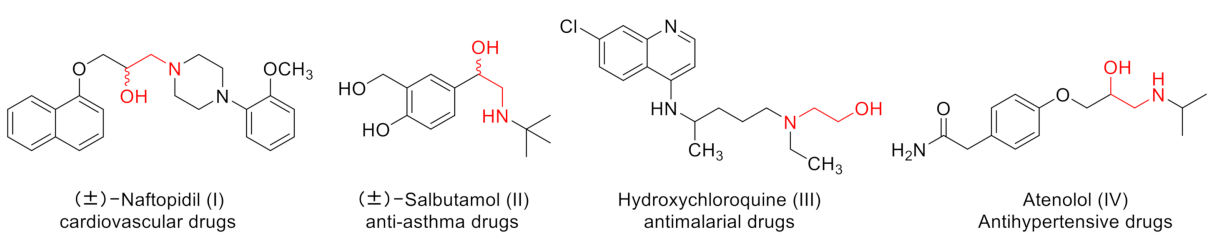

Figure 1. Examples of $\beta$-amino alcohol drugs.

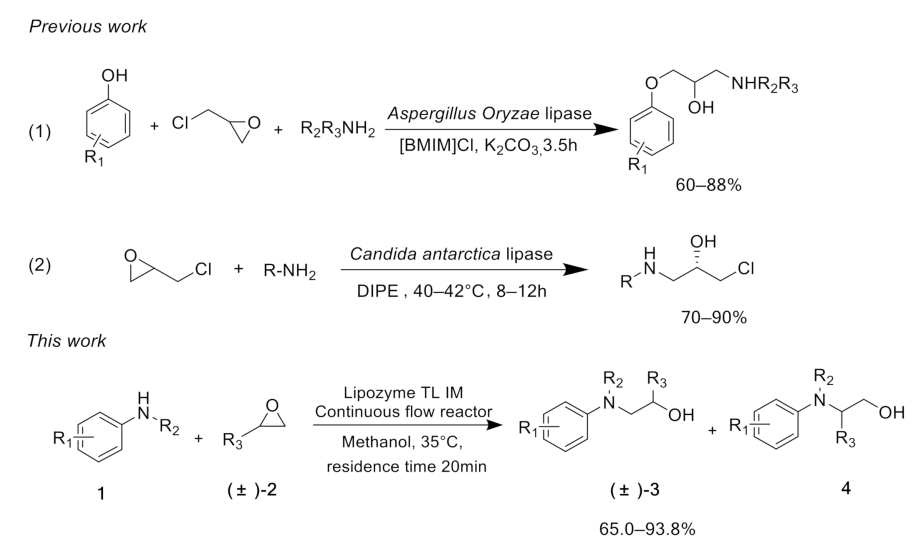

Scheme 1. Strategies for the preparation of $\beta$-amino alcohols by lipase.

Recently, continuous-flow technology has been widely used in enzymatic reactions, and the combination of biological catalytic technology and flow chemistry opens influential novel process windows [31-35]. With the advantages of high specific surface area, good heat transfer performance and efficient mixing, continuous-flow reactors can increase the reaction efficiency and selectivity [36-38]. To explore an efficient and novel synthesis protocol for $\beta$-amino alcohols, we first reported a continuous-flow method for the preparation of $\beta$-amino alcohol derivatives catalyzed by lipase from Thermomyces lanuginosus in continuous-flow reactors (Scheme 1). The purpose of our research is to investigate the effect of different reaction parameters on this enzymatic flow preparation method. We would like to rapidly establish relevant chemical compound libraries through this novel synthetic method for future pharmaceutical screening.

\section{Results and Discussion}

\subsection{Effect of Reaction Media and Catalyst}

First, we explored the enzymatic synthesis of $\beta$-amino alcohols by a ring-opening reaction in continuous-flow reactors (Table 1). We chose the reaction of epichlorohydrin with aniline as the model reaction to study (Scheme 2). For the $\beta$-amino alcohol synthesis reaction, the enzyme and the reaction medium are significant elements that influence the enzyme activity and reaction process. We conducted a blank control trial and found that this reaction could not proceed without the participation of lipase. Next, two different enzymes, Subtilisin and Lipozyme TL IM, were screened for the ring-opening of epichlorohydrin with aniline in different organic solvents. It was observed that the reaction could be promoted by increasing the polarity of the solvent. Lipozyme TL IM in methanol could catalyze the 
nucleophilic molecules to form the main product 3a with the best yield of $85.2 \%$ (Entry 2, Table 1). As a result, we synthesized the $\beta$-amino alcohols catalyzed by Lipozyme TL IM in methanol.

Table 1. Effect of reaction solvent and catalysts on the synthesis of $\beta$-amino alcohols under continuous-flow conditions ${ }^{a}$.

\begin{tabular}{cccccc}
\hline \multirow{2}{*}{ Entry } & \multirow{2}{*}{ Solvent } & Log $\mathbf{p}$ & Catalysts & \multicolumn{2}{c}{ Yield $^{\mathbf{b}} \mathbf{( \% )}$} \\
\cline { 5 - 6 } & & & & $\mathbf{3}$ & $\mathbf{4}$ \\
\hline 1 & Methanol & -0.76 & None & n.d. & n.d. \\
2 & Methanol & -0.76 & Lipozyme TL IM & $85.2 \pm 1.2$ & $3.1 \pm 1.1$ \\
3 & Ethanol & -0.24 & Lipozyme TL IM & $80.1 \pm 1.6$ & $2.9 \pm 1.2$ \\
4 & Acetonitrile & -0.33 & Lipozyme TL IM & $78.8 \pm 2.1$ & $1.8 \pm 1.5$ \\
5 & Toluene & 2.5 & Lipozyme TL IM & $76.2 \pm 0.8$ & $2.3 \pm 1.6$ \\
6 & n-Hexane & 3.94 & Lipozyme TL IM & $71.5 \pm 1.5$ & $1.7 \pm 1.2$ \\
7 & Methanol & -0.76 & Subtilisin & n.d. & n.d. \\
8 & Ethanol & -0.24 & Subtilisin & n.d. & n.d. \\
\hline
\end{tabular}

a Reaction conditions: continuous-flow reactor, feed 1, $10 \mathrm{~mL}$ solvent contained $5.0 \mathrm{mmol}$ aniline (1a); feed 2, $10 \mathrm{~mL}$ solvent contained $5.0 \mathrm{mmol}$ epichlorohydrin (2a), enzyme $870 \mathrm{mg}(174 \mathrm{mg} / \mathrm{mmol})$, flow rate $20.9 \mu \mathrm{L} \mathrm{min}{ }^{-1}$, residence time $30 \mathrm{~min}$, total reaction time $15.9 \mathrm{~h}, 40^{\circ} \mathrm{C}$. ${ }^{\mathrm{b}}$ Isolated yield. Yield: $100 \times$ (actual received quantity/ideal calculated quantity). The data are presented as average $\pm \mathrm{SD}$ of triplicate experiments.<smiles>Nc1ccccc1</smiles>

1a

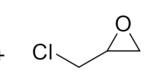

$( \pm)-2 a$<smiles>[Mg][Mg][Ba]</smiles>
Continuous flow reactor<smiles>OC(CCl)CNc1ccccc1</smiles>

$( \pm)-3 a$

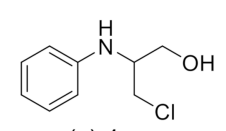

$( \pm)-4 a$

Scheme 2. The ring-opening of epichlorohydrin with aniline under continuous-flow conditions.

\subsection{Effect of Reaction Temperature}

Temperature has an important effect on the stability and catalytic activity of the enzyme [39]. In the interest of obtaining the optimum temperature, we adjusted it from 30 to $50{ }^{\circ} \mathrm{C}$ and the yield of products was determined at a different temperature with 30 min residence time. As shown in Figure 2, it is obvious that the yield increased from 30 to $35^{\circ} \mathrm{C}$. If the reaction temperature continued to increase, the yield decreased. Therefore, the optimum temperature of synthesizing $\beta$-amino alcohol is $35^{\circ} \mathrm{C}$.

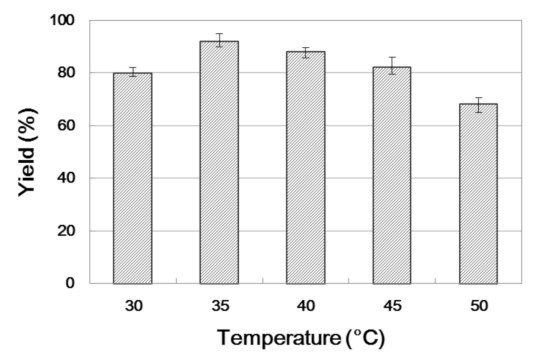

Figure 2. The influence of temperature on the enzymatic synthesis of $\beta$-amino alcohol 3a under continuous-flow conditions.

\subsection{Effect of Residence Time}

The residence time is an important parameter in the continuous processes because it describes the contact time between the reagents and the biocatalyst, which affects the conversion of the reaction. Hence, the influence of residence time on the reaction was studied by changing the residence time from 10 to $30 \mathrm{~min}$, and the results are shown in Figure 3. With the increase in residence time, interactions between the substrate and enzyme increased, which led to a higher yield of $\beta$-amino alcohol. After $20 \mathrm{~min}$, the optimum yield was obtained under a flow rate of $31.4 \mu \mathrm{L} \mathrm{min}{ }^{-1}$. Hence, $20 \mathrm{~min}$ was chosen as the best residence time for the subsequent study. 


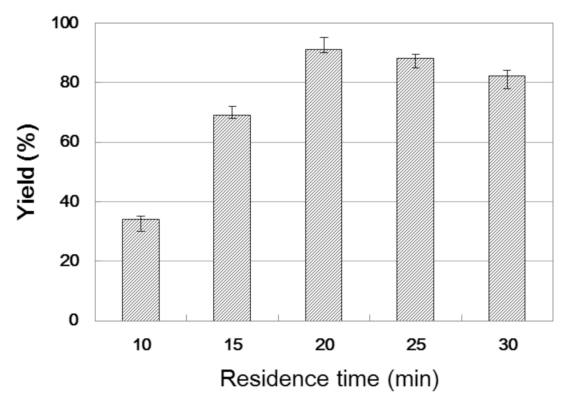

Figure 3. The influence of residence time on the enzymatic synthesis of $\beta$-amino alcohol 3a under continuous-flow conditions.

\subsection{Effect of Substrate Ratio}

In enzyme catalysis reaction, the relative content of substrates is the key to determine the composition of the final product and the efficiency of enzyme catalysis [40]. The influence of substrate ratio on reaction efficiency was studied by using different molar ratios of aniline to epichlorohydrin with a 30 min residence time (Figure 4). As the molar ratio of substrate increased, the catalytic efficiency of $\beta$-amino alcohol synthesis was improved. The highest yield of $91.3 \%$ was achieved when a molar ratio of aniline to epichlorohydrin of 1:1 was used. However, further changes in the molar ratio reduced the yield. Hence, aniline/epichlorohydrin $=1: 1$ is the optimal ratio for preparing $\beta$-amino alcohol.

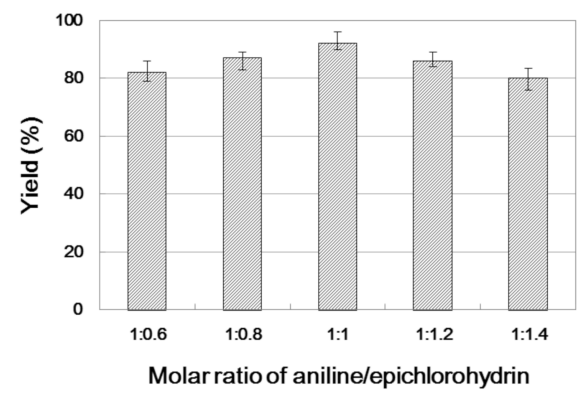

Figure 4. The influence of substrate ratio on the enzymatic synthesis of $\beta$-amino alcohol 3a under continuous-flow conditions.

\subsection{The Effect of Enzyme Reusability on the Reaction}

Enzyme reusability is a vital aspect of an enzymatic reaction due to its economic significance. The yield of $\beta$-amino alcohol 3a was examined over 10 reaction cycles to determine its reusability. As shown in Figure 5, an increase in the number of cycles is accompanied by a progressive decrease in yields, and a $43.4 \%$ yield was obtained during the tenth cycle. This result indicates that Lipozyme TL IM has sufficient reusability.

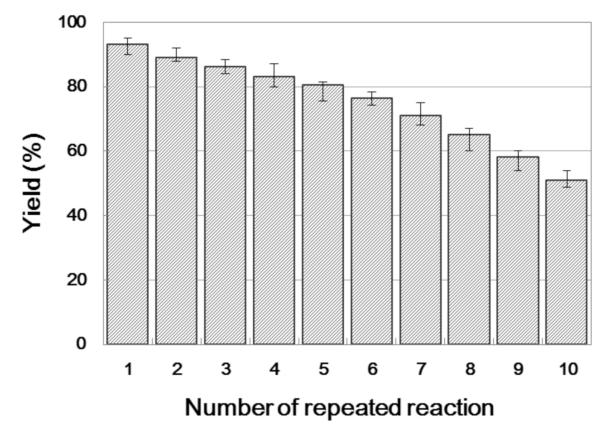

Figure 5. The influence of enzyme reusability on the enzymatic synthesis of $\beta$-amino alcohol 3a under continuous-flow conditions. 


\subsection{The Effect of Aromatic Amine Structure on the Reaction}

We further researched the influence of substrate structure on the synthesis of $\beta$-amino alcohols (Figure 6). As illustrated in Table 2, the yield of p-toluidine to epichlorohydrin was much higher $(92.7 \%$, entry 5$)$ than 4 -chloroaniline $(70.2 \%$, entry 6$)$ under the same conditions, which indicates that the introduction of an electron donor can effectively improve the reactivity of the amine, and the amines containing electron withdrawing groups such as halogen are not conducive to the ring-opening reaction. In addition, we also found that aniline $(91.3 \%$, entry 1$)$ has a better reactivity than $\mathrm{N}$-methylaniline (73.2\%, entry 2) because the steric hindrance of the secondary amine slowed down nucleophilic attack. Due to the steric effect, the amine was inclined to nucleophilic attack at the carbon atoms with less steric hindrance and the major product 3 was formed (Table 2). These results were confirmed by NMR.

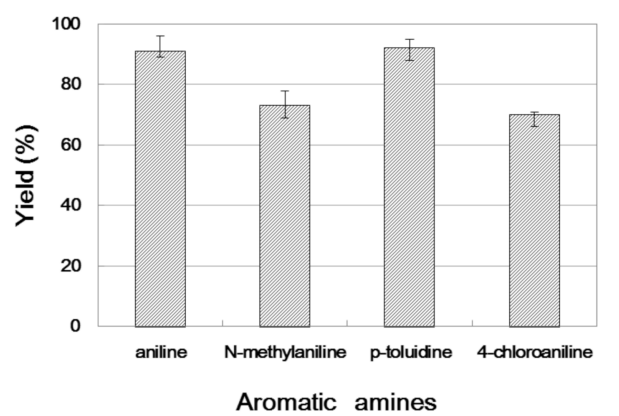

Figure 6. The influence of substrate structure on the enzymatic synthesis of $\beta$-amino alcohol 3a under continuous-flow conditions.

Table 2. Continuous-flow synthesis of $\beta$-amino alcohols catalyzed by Lipozyme TL IM. ${ }^{\text {a }}$

Entry (\%)


Table 2. Cont.

\begin{tabular}{|c|c|c|c|c|c|c|}
\hline \multirow{2}{*}{ Entry } & \multirow{2}{*}{ Amine } & \multirow{2}{*}{ Epoxide } & \multirow{2}{*}{ Major Product } & \multicolumn{2}{|c|}{ Yield $^{b}(\%)$} & \multirow{2}{*}{ ee $(\%)$} \\
\hline & & & & 3 & 4 & \\
\hline 7 & & & & $87.5 \pm 2.0$ & $4.1 \pm 0.8$ & 1 \\
\hline 8 & & & & $71.4 \pm 1.2$ & 0 & $\leq 1$ \\
\hline 9 & & & & $81.5 \pm 1.2$ & $4.7 \pm 0.9$ & $\leq 1$ \\
\hline 10 & & & & $89.8 \pm 1.5$ & $3.9 \pm 1.2$ & $\leq 1$ \\
\hline 11 & & & & $93.8 \pm 2.8$ & $3.2 \pm 0.7$ & $\leq 1$ \\
\hline 12 & & & & $76.5 \pm 1.6$ & $5.1 \pm 1.1$ & $\leq 1$ \\
\hline 13 & & & & $77.8 \pm 2.9$ & n.a. & 1 \\
\hline 14 & & & & $65.0 \pm 1.5$ & n.a. & $\leq 1$ \\
\hline 15 & & & & $68.5 \pm 1.1$ & n.a. & $\leq 1$ \\
\hline 16 & & & & $71.5 \pm 1.6$ & n.a. & $\leq 1$ \\
\hline 17 & & & & $75.6 \pm 1.8$ & n.a. & $\leq 1$ \\
\hline 18 & & & & $66.3 \pm 0.5$ & n.a. & $\leq 1$ \\
\hline
\end{tabular}

a Reaction conditions: continuous-flow reactors, feed 1,10 mL methanol contained $5.0 \mathrm{mmol}$ amine; feed 2, $10 \mathrm{~mL}$ methanol contained $5.0 \mathrm{mmol}$ epoxide, Lipozyme TL IM $870 \mathrm{mg}(174 \mathrm{mg} / \mathrm{mmol})$, flow rate $31.4 \mu \mathrm{L} \mathrm{min}{ }^{-1}$, residence time $20 \mathrm{~min}$, total reaction time $10.6 \mathrm{~h}, 35^{\circ} \mathrm{C}$. ${ }^{\mathrm{b}}$ Isolated yield. Yield: $100 \times$ (actual received quantity/ideal calculated quantity). The data are presented as average $\pm \mathrm{SD}$ of triplicate experiments. ${ }^{c}$ Enantiomeric excesses of 3 were determined by chiral HPLC analysis. 
Lastly, to study the applications of this innovative $\beta$-amino alcohol synthesis reaction catalyzed by lipase from Thermomyces lanuginosus in a continuous-flow reactor, six amines (aniline, N-methylaniline, o-toluidine, m-toluidine, p-toluidine, 4-chloroaniline) and three epoxides (epichlorohydrin, styrene oxide, cyclohexene oxide) were subjected to the optimized conditions (Scheme 3). Eighteen $\beta$-amino alcohol compounds were prepared in excellent yields by a ring-opening reaction catalyzed by Lipozyme TL IM under a continuous-flow reactor (Table 2).

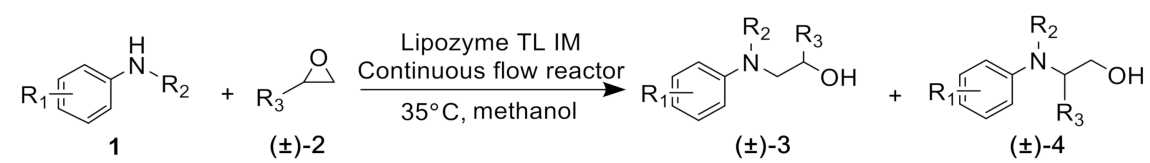

Scheme 3. The ring-opening of epoxides with amines under continuous-flow conditions.

\section{Materials and Methods}

\subsection{Materials}

Lipozyme TL IM from Thermomyces lanuginosus was purchased from Novo Nordisk. Subtilisin was purchased from Sigma. Aniline, $\mathrm{N}$-methylaniline, o-toluidine, m-toluidine, p-toluidine and 4-chloroaniline were purchased from Energy Chemical. ( \pm )-Epichlorohydrin, cyclohexene oxide and $( \pm)$-styrene oxide were purchased from Aladdin (Shanghai, China). Harvard Apparatus PHD 2000 syringe pumps were purchased from Harvard (Holliston, MA, USA). The flow reactor and the Y-mixer were made of perfluoroalkoxy (PFA) and purchased from Beijing Haigri Medical Engineering Design Co., Ltd. (Beijing, China). The products were analyzed by HPLC (LC-20AT, purchased from Shimadzu, Shanghai, China) with chiral columns (Chiralpak IF-3, Hexane/IPA solution 90/10 $(v / v)$, flow rate $=0.7 \mathrm{~mL} / \mathrm{min}$ ).

\subsection{Experimental Setup and Experiment Conditions}

The equipment configuration that was used for the synthesis of $\beta$-amino alcohols by a ring-opening reaction is shown in Figure 7 as well as in Figures S1 and S2. The experimental equipment consisted of a syringe pump (Harvard Apparatus PHD 2000), a substrate injector, a Y-shaped mixer, a flow reactor and a product collector. The flow reactor consisted of a $1 \mathrm{~m}$ PFA tube with an internal diameter of $2 \mathrm{~mm}$. The flow reaction tube was coiled and filled with Lipozyme TL IM (870 mg). Lipozyme TL IM was kept by using absorbent cotton at both ends of the flow reaction tube. We submerged the flow reactor in a water bath to regulate the temperature. $10 \mathrm{~mL}$ methanol contained $5.0 \mathrm{mmol}$ amines (feed $1,0.5 \mathrm{M}$ ) and $10 \mathrm{~mL}$ methanol contained 5.0 mmol epoxides (feed 2, $0.5 \mathrm{M}$ ). Streams 1 and 2 were mixed together in a Y-mixer and the mixture stream $\left(31.4 \mu \mathrm{L} \mathrm{min}^{-1}\right)$ was passed through the flow reactor. The residence time was $20 \mathrm{~min}$ at $35^{\circ} \mathrm{C}$ and the solution was collected in a glass vessel and evaporated. The product was isolated by silica gel chromatography (the mobile phase petroleum ether/ethyl acetate from $15 / 1$ to $12 / 1$ ). The major products were determined by ${ }^{1} \mathrm{H}$ NMR and ${ }^{13} \mathrm{C} N M R$.

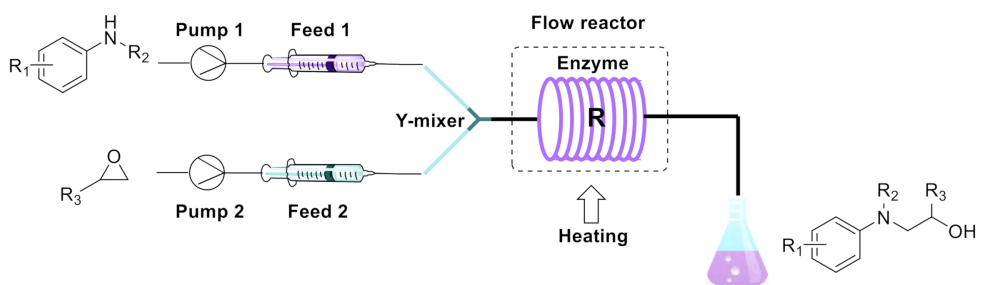

Figure 7. Equipment for the enzymatic synthesis of $\beta$-amino alcohols under continuous-flow conditions. 


\subsection{Analytical Methods}

\subsubsection{Thin-Layer Chromatography (TLC)}

TLC analysis with ethyl acetate/petroleum ether $1 / 4(v / v)$ as the eluent. The results were detected by UV irradiation at $254 \mathrm{~nm}$.

\subsubsection{Nuclear Magnetic Resonance (NMR)}

The products were characterized by ${ }^{1} \mathrm{H}$ NMR and ${ }^{13} \mathrm{C}$ NMR. ${ }^{1} \mathrm{H}(500 \mathrm{MHz})$ and ${ }^{13} \mathrm{C}(126 \mathrm{MHz})$ NMR spectra were obtained on a Bruker-AVANCE III $500 \mathrm{MHz}$ NMR spectrometer. The sample was dissolved in $\mathrm{CDCl}_{3}$ or DMSO- $d_{6}$ and with $22{ }^{\circ} \mathrm{C}$ temperature.

1-Chloro-3-(phenylamino)propan-2-ol (3a). Yellow oil. 0.845 g. ${ }^{1} \mathrm{H}$ NMR $\left(500 \mathrm{MHz}, \mathrm{DMSO}-d_{6}\right): \delta$ 7.16-7.06 (m, 2H), 6.71-6.62 (m, 2H), $6.58(\mathrm{tt}, J=7.3,1.1 \mathrm{~Hz}, 1 \mathrm{H}), 5.42(\mathrm{~s}, 2 \mathrm{H}), 3.97-3.87(\mathrm{~m}, 1 \mathrm{H}), 3.73$ $(\mathrm{dd}, J=11.1,4.5 \mathrm{~Hz}, 1 \mathrm{H}), 3.64(\mathrm{dd}, J=11.1,5.7 \mathrm{~Hz}, 1 \mathrm{H}), 3.24(\mathrm{dd}, J=13.2,5.6 \mathrm{~Hz}, 1 \mathrm{H}), 3.10(\mathrm{dd}, J=13.1$, $6.3 \mathrm{~Hz}, 1 \mathrm{H}) ;{ }^{13} \mathrm{C}$ NMR $\left(126 \mathrm{MHz}, \mathrm{CDCl}_{3}\right): \delta 147.33,129.42,118.65,113.66,69.71,47.55,47.41$.

1-Chloro-3-(methyl(phenyl)amino)propan-2-ol (3b). Yellow oil. 0.728 g. ${ }^{1} \mathrm{H}$ NMR $(500 \mathrm{MHz}$, DMSO- $\left.d_{6}\right): \delta 7.23-7.16(\mathrm{~m}, 2 \mathrm{H}), 6.80-6.71(\mathrm{~m}, 2 \mathrm{H}), 6.65(\mathrm{tt}, J=7.2,1.0 \mathrm{~Hz}, 1 \mathrm{H}), 5.37(\mathrm{~d}, J=4.0 \mathrm{~Hz}, 1 \mathrm{H})$, $3.99(\mathrm{~s}, 1 \mathrm{H}), 3.67(\mathrm{dd}, J=11.1,4.5 \mathrm{~Hz}, 1 \mathrm{H}), 3.61(\mathrm{dd}, J=11.1,5.5 \mathrm{~Hz}, 1 \mathrm{H}), 3.52(\mathrm{dd}, J=14.9,5.3 \mathrm{~Hz}, 1 \mathrm{H})$, $3.32(\mathrm{dd}, J=14.9,7.0 \mathrm{~Hz}, 1 \mathrm{H}), 2.97(\mathrm{~s}, 3 \mathrm{H}) ;{ }^{13} \mathrm{C}$ NMR $\left(126 \mathrm{MHz}, \mathrm{DMSO}-d_{6}\right): \delta 129.35,117.88,113.18$, $68.98,56.77,47.71,39.76$.

1-Chloro-3-(o-tolylamino)propan-2-ol (3c). Yellow oil. 0.821 g. ${ }^{1} \mathrm{H}$ NMR $\left(500 \mathrm{MHz}, \mathrm{DMSO}-d_{6}\right) \delta$ 7.20-7.07 (m, 2H), 6.78-6.68 (m, 2H), $5.39(\mathrm{~d}, J=6.3 \mathrm{~Hz}, 2 \mathrm{H}), 4.20-4.13(\mathrm{~m}, 1 \mathrm{H}), 3.77-3.64(\mathrm{~m}, 2 \mathrm{H}), 3.46$ $(\mathrm{dd}, J=4.4,13.1 \mathrm{~Hz}, 1 \mathrm{H}), 3.30(\mathrm{dd}, J=7.3,13.1 \mathrm{~Hz}, 1 \mathrm{H}), 2.20(\mathrm{~s}, 3 \mathrm{H}) ;{ }^{13} \mathrm{C}$ NMR $\left(126 \mathrm{MHz}, \mathrm{DMSO}-d_{6}\right): \delta$ 145.3, 130.4, 127.2, 123.0, 118.3, 110.6, 69.7, 47.8, 47.4, 17.5.

1-Chloro-3-(m-tolylamino)propan-2-ol (3d). Yellow oil. 0.882 g. ${ }^{1} \mathrm{H}$ NMR (500 MHz, DMSO- $\left.d_{6}\right): \delta$ $7.14(1 \mathrm{H}, \mathrm{s}), 6.39-6.60(3 \mathrm{H}, \mathrm{m}), 5.35(\mathrm{~d}, J=6.1 \mathrm{~Hz}, 2 \mathrm{H}), 3.79(\mathrm{~d}, J=7.1 \mathrm{~Hz}, 1 \mathrm{H}), 3.69(\mathrm{dd}, J=12.2,4.4 \mathrm{~Hz}$, $1 \mathrm{H}), 3.59(\mathrm{dd}, J=12.2,5.8 \mathrm{~Hz}, 1 \mathrm{H}), 3.08(\mathrm{dd}, J=12.9,5.8 \mathrm{~Hz}, 1 \mathrm{H}), 3.00(\mathrm{dd}, J=12.9,5.8 \mathrm{~Hz}, 1 \mathrm{H}), 2.15(\mathrm{~s}$, $3 \mathrm{H}) ;{ }^{13} \mathrm{C}$ NMR $\left(126 \mathrm{MHz}\right.$, DMSO- $\left.d_{6}\right): \delta 147.5,139.2,129.4,117.4,113.2,110.5,76.2,50.8,48.9,21.3$.

1-Chloro-3-(p-tolylamino)propan-2-ol (3e). White powder. 0.922 g. ${ }^{1} \mathrm{H}$ NMR (500 MHz, DMSO-d $\left.d_{6}\right): \delta$ 6.99-6.82 (m, 2H), 6.59-6.44 (m, 2H), $5.31(\mathrm{~d}, J=5.5 \mathrm{~Hz}, 2 \mathrm{H}), 3.82(\mathrm{~d}, J=7.1 \mathrm{~Hz}, 1 \mathrm{H}), 3.69(\mathrm{dd}, J=11.1$, $4.4 \mathrm{~Hz}, 1 \mathrm{H}), 3.59(\mathrm{dd}, J=11.1,5.7 \mathrm{~Hz}, 1 \mathrm{H}), 3.14(\mathrm{dd}, J=13.1,5.8 \mathrm{~Hz}, 1 \mathrm{H}), 3.00(\mathrm{dd}, J=13.1,6.2 \mathrm{~Hz}, 1 \mathrm{H})$, $2.15(\mathrm{~s}, 3 \mathrm{H}) ;{ }^{13} \mathrm{C} \mathrm{NMR}\left(126 \mathrm{MHz}, \mathrm{CDCl}_{3}\right): \delta 144.87,129.92,128.23,114.00,69.73,48.01,47.65,20.41$.

1-Chloro-3-((4-chlorophenyl)amino)propan-2-ol (3f). Yellow oil. 0.772 g. ${ }^{1} \mathrm{H} \mathrm{NMR}(500 \mathrm{MHz}$, DMSO- $\left.d_{6}\right) \delta 7.09(\mathrm{~d}, J=8.9 \mathrm{~Hz}, 2 \mathrm{H}), 6.62(\mathrm{~d}, J=8.9 \mathrm{~Hz}, 2 \mathrm{H}), 5.77(\mathrm{~s}, 1 \mathrm{H}), 5.38(\mathrm{~s}, 1 \mathrm{H}), 3.90-3.80(\mathrm{~m}, 1 \mathrm{H})$, $3.69(\mathrm{dd}, J=11.1,4.5 \mathrm{~Hz}, 1 \mathrm{H}), 3.60(\mathrm{dd}, J=11.1,5.6 \mathrm{~Hz}, 1 \mathrm{H}), 3.18(\mathrm{dd}, J=13.3,5.5 \mathrm{~Hz}, 1 \mathrm{H}), 3.04(\mathrm{dd}$, $J=13.3,6.3 \mathrm{~Hz}, 1 \mathrm{H}) ;{ }^{13} \mathrm{C}$ NMR $\left(126 \mathrm{MHz}, \mathrm{CDCl}_{3}\right): \delta 146.32,129.18,122.84,114.44,69.80,47.56,47.22$.

1-Phenyl-2-(phenylamino)ethan-1-ol (3g). Yellow oil. 0.932 g. ${ }^{1} \mathrm{H}$ NMR (500 MHz, DMSO-d 6 ): $\delta$ $7.49-7.43(\mathrm{~m}, 2 \mathrm{H}), 7.33(\mathrm{t}, J=7.6 \mathrm{~Hz}, 2 \mathrm{H}), 7.23(\mathrm{t}, J=7.3 \mathrm{~Hz}, 1 \mathrm{H}), 7.04(\mathrm{dd}, J=8.5,7.2 \mathrm{~Hz}, 2 \mathrm{H}), 6.60(\mathrm{~d}$, $J=7.4 \mathrm{~Hz}, 2 \mathrm{H}), 6.54(\mathrm{t}, J=7.2 \mathrm{~Hz}, 1 \mathrm{H}), 5.99(\mathrm{~d}, J=5.9 \mathrm{~Hz}, 1 \mathrm{H}), 5.07(\mathrm{t}, J=5.8 \mathrm{~Hz}, 1 \mathrm{H}), 4.48-4.42(\mathrm{~m}$, $1 \mathrm{H}), 3.77-3.63(\mathrm{~m}, 2 \mathrm{H}) ;{ }^{13} \mathrm{C} \mathrm{NMR}\left(126 \mathrm{MHz}, \mathrm{CDCl}_{3}\right): \delta 143.56,137.60,129.25,128.86,128.17,127.42$, $120.92,116.69,66.14,62.97$.

2-(Methyl(phenyl)amino)-1-phenylethan-1-ol (3h). Yellow oil. 0.810 g. ${ }^{1} \mathrm{H}$ NMR (500 MHz, DMSO- $\left.d_{6}\right)$ : $\delta 7.32(\mathrm{t}, J=7.6 \mathrm{~Hz}, 2 \mathrm{H}), 7.28(\mathrm{~d}, J=7.3 \mathrm{~Hz}, 2 \mathrm{H}), 7.26-7.21(\mathrm{~m}, 1 \mathrm{H}), 7.15(\mathrm{dd}, J=8.8,7.2 \mathrm{~Hz}, 2 \mathrm{H}), 6.78(\mathrm{~d}$, $J=8.2 \mathrm{~Hz}, 2 \mathrm{H}), 6.61(\mathrm{t}, J=7.2 \mathrm{~Hz}, 1 \mathrm{H}), 4.98-4.92(\mathrm{~m}, 1 \mathrm{H}), 4.89(\mathrm{~s}, 1 \mathrm{H}), 3.91(\mathrm{qd}, J=11.4,6.9 \mathrm{~Hz}, 2 \mathrm{H})$, $2.82(\mathrm{~s}, 3 \mathrm{H}) ;{ }^{13} \mathrm{C}$ NMR $\left(126 \mathrm{MHz}, \mathrm{DMSO}-d_{6}\right): \delta 150.14,139.99,128.84,128.26,127.19,126.81,115.76$, $112.31,62.92,61.81,32.20$.

1-Phenyl-2-(o-tolylamino)ethan-1-ol (3i). Yellow oil. 0.925 g. ${ }^{1} \mathrm{H}$ NMR $\left(500 \mathrm{MHz}, \mathrm{DMSO}-d_{6}\right): \delta$ 7.76-7.38 (m, 4H), 7.36-7.31 (m, 1H), 7.17-7.12 (m, 1H), 7.10-7.06 (m, 1H), 6.74-6.68 (m, 2H), $5.63(\mathrm{~d}$, $J=5.9 \mathrm{~Hz}, 1 \mathrm{H}), 4.98(\mathrm{t}, J=6.2 \mathrm{~Hz}, 1 \mathrm{H}), 3.48(\mathrm{dd}, J=5.9,4.0 \mathrm{~Hz}, 2 \mathrm{H}), 3.36(\mathrm{dt}, J=6.2,4.0 \mathrm{~Hz}, 1 \mathrm{H}), 2.15(\mathrm{~s}$, $3 \mathrm{H}) ;{ }^{13} \mathrm{C}$ NMR (126 MHz, DMSO- $\left.d_{6}\right): \delta 145.9,142.2,130.4,128.8,128.2,127.3,126.0,122.9,117.8,110.5$, 72.6, 51.8, 17.6 . 
1-Phenyl-2-(m-tolylamino)ethan-1-ol (3j). Yellow oil. 1.019 g. ${ }^{1} \mathrm{H}$ NMR (500 MHz, DMSO- $\left.d_{6}\right): \delta$ $7.45-7.39(\mathrm{~m}, 2 \mathrm{H}), 7.36(\mathrm{dd}, J=8.3,6.9 \mathrm{~Hz}, 2 \mathrm{H}), 7.25-7.16(\mathrm{~m}, 1 \mathrm{H}), 6.95(\mathrm{dd}, J=8.5,7.2 \mathrm{~Hz}, 1 \mathrm{H}), 6.43(\mathrm{~s}$, $1 \mathrm{H}), 6.35-6.39(\mathrm{~m}, 2 \mathrm{H}), 6.01(\mathrm{~d}, J=6.1 \mathrm{~Hz}, 1 \mathrm{H}), 5.09(\mathrm{t}, J=6.01 \mathrm{~Hz}, 1 \mathrm{H}), 4.48-4.42(\mathrm{~m}, 1 \mathrm{H}), 3.77-3.63(\mathrm{~m}$, 2H), $2.11(\mathrm{~s}, 3 \mathrm{H}) ;{ }^{13} \mathrm{C}$ NMR (126 MHz, DMSO- $\left.d_{6}\right): \delta 145.8,140.2,138.9,129.8,129.5,127.6,127.0,121.5$, $116.9,110.9,66.3,61.5,22.2$.

1-Phenyl-2-(p-tolylamino)ethan-1-ol (3k). Yellow oil. 1.065 g. ${ }^{1} \mathrm{H}$ NMR $\left(500 \mathrm{MHz}, \mathrm{DMSO}-d_{6}\right): \delta$ $7.43-7.38(\mathrm{~m}, 2 \mathrm{H}), 7.30(\mathrm{dd}, J=8.3,6.9 \mathrm{~Hz}, 2 \mathrm{H}), 7.25-7.16(\mathrm{~m}, 1 \mathrm{H}), 6.82(\mathrm{~d}, J=8.3 \mathrm{~Hz}, 2 \mathrm{H}), 6.47(\mathrm{~d}$, $J=8.4 \mathrm{~Hz}, 2 \mathrm{H}), 5.73(\mathrm{~d}, J=5.8 \mathrm{~Hz}, 1 \mathrm{H}), 4.99(\mathrm{t}, J=5.8 \mathrm{~Hz}, 1 \mathrm{H}), 4.36(\mathrm{dt}, J=7.2,5.0 \mathrm{~Hz}, 1 \mathrm{H}), 3.71-3.55$ $(\mathrm{m}, 2 \mathrm{H}), 2.11(\mathrm{~s}, 3 \mathrm{H}) ;{ }^{13} \mathrm{C}$ NMR $\left(126 \mathrm{MHz}, \mathrm{DMSO}-d_{6}\right): \delta 145.87,142.25,129.13,128.12,126.95,126.68$, $124.25,113.09,66.24,59.92,20.04$.

2-((4-Chlorophenyl)amino)-1-phenylethan-1-ol (31). Clear oil. 0.945 g. ${ }^{1} \mathrm{H}$ NMR (500 MHz, DMSO- $\left.d_{6}\right)$ : $\delta 7.40-7.33(\mathrm{~m}, 2 \mathrm{H}), 7.30(\mathrm{dd}, J=8.4,6.8 \mathrm{~Hz}, 2 \mathrm{H}), 7.25-7.18(\mathrm{~m}, 1 \mathrm{H}), 7.00(\mathrm{~d}, J=8.8 \mathrm{~Hz}, 2 \mathrm{H}), 6.52(\mathrm{~d}$, $J=8.9 \mathrm{~Hz}, 2 \mathrm{H}), 6.22(\mathrm{~d}, J=6.5 \mathrm{~Hz}, 1 \mathrm{H}), 4.96(\mathrm{~s}, 1 \mathrm{H}), 4.34(\mathrm{td}, J=6.9,5.1 \mathrm{~Hz}, 1 \mathrm{H}), 3.59(\mathrm{dd}, J=12.3,6.2$ $\mathrm{Hz}, 2 \mathrm{H}) ;{ }^{13} \mathrm{C}$ NMR $\left(126 \mathrm{MHz}\right.$, DMSO- $\left.d_{6}\right): \delta 147.02,141.60,128.32,128.17,126.88,126.80,118.98,114.10$, $65.96,59.56$.

2-(Phenylamino)cyclohexan-1-ol (3m). White powder. 0.743 g. ${ }^{1} \mathrm{H}$ NMR (500 MHz, DMSO- $\left.d_{6}\right): \delta$ $7.07-7.00(\mathrm{~m}, 2 \mathrm{H}), 6.59(\mathrm{~d}, J=7.6 \mathrm{~Hz}, 2 \mathrm{H}), 6.47(\mathrm{t}, J=7.2 \mathrm{~Hz}, 1 \mathrm{H}), 5.22(\mathrm{~d}, J=6.9 \mathrm{~Hz}, 1 \mathrm{H}), 4.55(\mathrm{~d}, J=4.7$ $\mathrm{Hz}, 1 \mathrm{H}), 2.98(\mathrm{~s}, 1 \mathrm{H}), 1.98(\mathrm{dd}, J=12.3,3.6 \mathrm{~Hz}, 1 \mathrm{H}), 1.90-1.84(\mathrm{~m}, 1 \mathrm{H}), 1.68-1.55(\mathrm{~m}, 2 \mathrm{H}), 1.36-1.19(\mathrm{~m}$, $4 \mathrm{H}), 1.12-1.03(\mathrm{~m}, 1 \mathrm{H}) ;{ }^{13} \mathrm{C}$ NMR $\left(126 \mathrm{MHz}, \mathrm{CDCl}_{3}\right): \delta 147.01,129.34,118.94,114.93,74.23,60.63,33.21$, 31.30, 24.94, 24.23 .

2-(Methyl(phenyl)amino)cyclohexan-1-ol (3n). Yellow oil. 0.666 g. ${ }^{1} \mathrm{H}$ NMR (500 MHz, DMSO- $\left.d_{6}\right): \delta$ 7.20-7.07 (m, 2H), $6.77(\mathrm{dd}, J=8.6,2.3 \mathrm{~Hz}, 2 \mathrm{H}), 6.58(\mathrm{dt}, J=6.4,5.8,3.4 \mathrm{~Hz}, 1 \mathrm{H}), 4.49-4.41(\mathrm{~m}, 1 \mathrm{H})$, $3.61-3.50(\mathrm{~m}, 1 \mathrm{H}), 3.44-3.36(\mathrm{~m}, 1 \mathrm{H}), 2.79-2.69(\mathrm{~m}, 3 \mathrm{H}), 2.00-1.93(\mathrm{~m}, 1 \mathrm{H}), 1.70-1.58(\mathrm{~m}, 3 \mathrm{H}), 1.41$ (qd, $J=12.2,3.2 \mathrm{~Hz}, 1 \mathrm{H}), 1.34-1.21(\mathrm{~m}, 3 \mathrm{H}) ;{ }^{13} \mathrm{C} \mathrm{NMR}\left(126 \mathrm{MHz}, \mathrm{DMSO}-d_{6}\right): \delta 150.78,128.66,115.24,112.62$, $69.21,63.74,35.15,30.46,27.45,24.94,24.28$.

2-(o-Tolylamino)cyclohexan-1-ol (3o). Colorless oil. 0.702 g. ${ }^{1} \mathrm{H}$ NMR (500 MHz, DMSO- $\left.d_{6}\right): \delta$ 7.26-7.06 (m, 2H), 6.80-6.68 (m, 2H), $5.32(\mathrm{~d}, J=7.1 \mathrm{~Hz}, 1 \mathrm{H}), 4.27(\mathrm{~d}, J=4.7 \mathrm{~Hz}, 1 \mathrm{H}), 3.24-3.18(\mathrm{~m}$, $1 \mathrm{H}), 2.74(\mathrm{ddt}, J=12.3,7.6,4.2 \mathrm{~Hz}, 1 \mathrm{H}), 2.16(\mathrm{~s}, 3 \mathrm{H}), 2.13(\mathrm{~s}, 2 \mathrm{H}), 1.79-1.72(\mathrm{~m}, 2 \mathrm{H}), 1.45-1.29(\mathrm{~m}, 3 \mathrm{H})$, $1.09-1.02(\mathrm{~m}, 1 \mathrm{H}) ;{ }^{13} \mathrm{C}$ NMR $\left(126 \mathrm{MHz}\right.$, DMSO- $\left.d_{6}\right) \delta 145.90,130.66,127.39,123.35,118.14,111.85,74.96$, $60.12,33.43,32.09,25.27,24.51,17.90$.

2-(m-Tolylamino)cyclohexan-1-ol (3p). Colorless oil. 0.733 g. ${ }^{1} \mathrm{H}$ NMR (500 MHz, DMSO- $\left.d_{6}\right): \delta 7.02$ $(\mathrm{s}, 1 \mathrm{H}), 6.39-6.60(\mathrm{~m}, 3 \mathrm{H}), 4.59(\mathrm{t}, J=3.6 \mathrm{~Hz}, 1 \mathrm{H}), 3.43-3.33(\mathrm{~m}, 1 \mathrm{H}), 3.19-3.27(\mathrm{~m}, 1 \mathrm{H}), 3.02-3.08(\mathrm{~m}$, 1H) $2.21(\mathrm{~s}, 3 \mathrm{H}), 2.00-2.05(\mathrm{~m}, 2 \mathrm{H}), 1.61-1.69(\mathrm{~m}, 2 \mathrm{H}), 1.17-1.35 \mathrm{ppm}(\mathrm{m}, 4 \mathrm{H}) ;{ }^{13} \mathrm{C}$ NMR $(126 \mathrm{MHz}$, DMSO- $\left.d_{6}\right): \delta 21.5,23.7,25.0,31.0,33.4,59.9,75.6,111.5,115.6,118.5,128.7,139.0,148.0$.

2-(p-Tolylamino)cyclohexan-1-ol (3q). Yellow oil. 0.775 g. ${ }^{1} \mathrm{H}$ NMR $\left(500 \mathrm{MHz}, \mathrm{DMSO}-d_{6}\right): \delta 6.90(\mathrm{~d}$, $J=8.2 \mathrm{~Hz}, 2 \mathrm{H}), 6.57(\mathrm{~d}, J=8.4 \mathrm{~Hz}, 2 \mathrm{H}), 4.97(\mathrm{~s}, 1 \mathrm{H}), 4.59(\mathrm{t}, J=3.6 \mathrm{~Hz}, 1 \mathrm{H}), 3.43-3.33(\mathrm{~m}, 1 \mathrm{H}), 3.00(\mathrm{~s}$, $1 \mathrm{H}), 2.19(\mathrm{~s}, 3 \mathrm{H}), 2.11-2.00(\mathrm{~m}, 1 \mathrm{H}), 1.99-1.90(\mathrm{~m}, 1 \mathrm{H}), 1.66(\mathrm{ddd}, J=32.4,8.3,3.8 \mathrm{~Hz}, 2 \mathrm{H}), 1.39-1.25(\mathrm{~m}$, $3 \mathrm{H}), 1.14-1.03(\mathrm{~m}, 1 \mathrm{H}) ;{ }^{13} \mathrm{C}$ NMR $\left(126 \mathrm{MHz}\right.$, DMSO- $\left.d_{6}\right): \delta 146.52,129.22,123.70,112.75,71.81,58.28$, $33.64,30.58,23.88,23.87,20.09$.

2-((4-Chlorophenyl)amino)cyclohexan-1-ol (3r). White solid. 0.746 g. ${ }^{1} \mathrm{H}$ NMR (500 MHz, DMSO- $\left.d_{6}\right)$ : $\delta 7.04(\mathrm{~d}, J=8.9 \mathrm{~Hz}, 2 \mathrm{H}), 6.59(\mathrm{~d}, J=8.9 \mathrm{~Hz}, 2 \mathrm{H}), 5.49(\mathrm{~d}, J=7.1 \mathrm{~Hz}, 1 \mathrm{H}), 4.57(\mathrm{~d}, J=4.7 \mathrm{~Hz}, 1 \mathrm{H}), 2.96$ $(\mathrm{ddt}, J=11.6,7.8,3.9 \mathrm{~Hz}, 1 \mathrm{H}), 1.94(\mathrm{dd}, J=13.3,4.0 \mathrm{~Hz}, 1 \mathrm{H}), 1.86(\mathrm{dd}, J=9.6,5.3 \mathrm{~Hz}, 1 \mathrm{H}), 1.68-1.55$ $(\mathrm{m}, 2 \mathrm{H}), 1.32-1.21(\mathrm{~m}, 4 \mathrm{H}), 1.07(\mathrm{t}, J=11.0 \mathrm{~Hz}, 1 \mathrm{H}) ;{ }^{13} \mathrm{C}$ NMR $\left(126 \mathrm{MHz}, \mathrm{DMSO}-d_{6}\right): \delta 147.76,128.35$, $118.03,113.58,71.80,57.67,33.66,30.32,23.74,23.65$.

\section{Conclusions}

In conclusion, we presented an attractive method for the synthesis of $\beta$-amino alcohols in a continuous-flow reactor. Lipozyme TL IM from Thermomyces lanuginosus was first used in the $\beta$-amino alcohol synthesis reaction. The combination of an environmentally friendly biocatalyst and continuous-flow technology displayed high efficiency in our research. We explored the influences of 
reaction medium, temperature, substrate ratio, residence time and reactant structure on the ring-opening reaction. Using this method, $18 \beta$-amino alcohols were quickly prepared. The striking characteristics of this method include the green reaction medium (methanol), short period of residence time (20 min), mild reaction temperature $\left(35^{\circ} \mathrm{C}\right)$ and the flexible and easy to control biocatalysis reaction process, which blaze a new road for the synthesis of $\beta$-amino alcohols. The use of continuous-flow technology to prepare $\beta$-amino alcohol derivatives is an innovation that allows the application of enzyme reactors in the biotransformation of $\beta$-amino alcohol derivatives.

Supplementary Materials: The following are available online at http://www.mdpi.com/2073-4344/10/12/1419/s1, Figure S1: Syringe pump and reactant injector., Figure S2: Photograph of the continuous flow system. Figure S3: Proposed mechanism of lipase catalyzed ring-opening reaction of epoxide.

Author Contributions: M.X. and L.-H.D. conceptualized the studies, carried out data collection, data analysis, drafted and revised the manuscript; M.-J.Y., Y.P. and L.-Y.Z. conceptualized the studies, carried out data collection, drafted and revised the manuscript; M.X., L.-H.D. and X.-P.L. conceptualized the studies and revised the manuscript. M.X. and Z.-M.O. analyzed the data and revised the manuscript. All authors gave final approval for publication. All authors have read and agreed to the published version of the manuscript.

Funding: This research was funded by the Natural Science Foundation of Zhejiang Province grant number [LGN20C200020], the Key Research \& Development Projects of Zhejiang Province grant number [2020C03090], the Zhejiang Provincial Key Discipline of Chemistry Biology, the National Science and Technology Support Project [2015BAD14B0305], the National Natural Science Foundation of China [21306172], the Science and Technology Research Program of Zhejiang Province grant number [2014C32094] and the APC was funded by the Natural Science Foundation of Zhejiang University of Technology grant number [116004029].

Acknowledgments: We thank the Natural Science Foundation of Zhejiang Province and the Key Research and Development Projects of Zhejiang Province (LGN20C200020 and 2020C03090), the Zhejiang Provincial Key Discipline of Chemistry Biology, the National Science and Technology Support Project (2015BAD14B0305), the National Natural Science Foundation of China (21306172), the Science and Technology Research Program of Zhejiang Province (2014C32094) as well as the Natural Science Foundation of Zhejiang University of Technology (116004029) for financial support.

Conflicts of Interest: The authors declare no conflict of interest.

\section{References}

1. Mohebbi, M.; Salehi, P.; Bararjanian, M.; Ebrahimi, S.N. Noscapine-derived $\beta$-amino alcohols as new organocatalysts for enantioselective addition of diethylzinc to aldehydes. J. Iran. Chem. Soc. 2017, 15, 47-53. [CrossRef]

2. Castejón, P.; Moyano, A.; Pericàs, M.A.; Riera, A. Access to stereodefined $\beta$-hydroxy- $\gamma$-amino acids. Enantioselective synthesis of fully protected cyclohexylstatine. Tetrahedron 1996, 52, 7063-7086. [CrossRef]

3. Alonso, D.A.; Guijarro, D.; Pinho, P.; Temme, O.; Andersson, P.G. (1S,3R,4R)-2-azanorbornylmethanol, an efficient ligand for ruthenium-catalyzed asymmetric transfer hydrogenation of ketones. J. Org. Chem. 1998, 63, 2749-2751. [CrossRef] [PubMed]

4. Ager, D.J.; Prakash, I.; Schaad, D.R. 1,2-Amino alcohols and their heterocyclic derivatives as chiral auxiliaries in asymmetric synthesis. Chem. Rev. 1996, 96, 835-875. [CrossRef] [PubMed]

5. Nicolaou, K.C.; Boddy, C.N. Atropselective macrocyclization of diaryl ether ring systems: Application to the synthesis of vancomycin model systems. J. Am. Chem. Soc. 2002, 124, 10451-10455. [CrossRef] [PubMed]

6. Deyrup, J.A.; Moye, C.L. 1,2,3-Oxathiazolidines-A new heterocyclic system. J. Org. Chem. 1969, 34, $175-179$. [CrossRef]

7. Yamada, S.; Ohkura, T.; Uchida, S.; Inabe, K.; Iwatani, Y.; Kimura, R.; Hoshino, T.; Kaburagi, T. A sustained increase in $\beta$-adrenoceptors during long-term therapy with metoprolol and bisoprolol in patients with heart failure from idiopathic dilated cardiomyopathy. Life Sci. 1996, 58, 1737-1744. [CrossRef]

8. Khirani, S.; Dabaj, I.; Amaddeo, A.; Olmo Arroyo, J.; Ropers, J.; Tirolien, S.; Coudert, V.; Estournet, B.; Fauroux, B.; Quijano-Roy, S. Effect of Salbutamol on Respiratory Muscle Strength in Spinal Muscular Atrophy. Pediatr. Neurol. 2017, 73, 78-87. [CrossRef] [PubMed]

9. Zhu, S.; Meng, L.; Zhang, Q.; Wei, L. Synthesis and evaluation of febrifugine analogues as potential antimalarial agents. Bioorg. Med. Chem. Lett. 2006, 16, 1854-1858. [CrossRef] 
10. Stahlberg, J.; Henriksson, H.; Divne, C.; Isaksson, R.; Pettersson, G.; Johansson, G.; Jones, T.A. Structural basis for enantiomer binding and separation of a common beta-blocker: Crystal structure of cellobiohydrolase Cel7A with bound (S)-propranolol at 1.9 A resolution. J. Mol. Biol. 2001, 305, 79-93. [CrossRef]

11. Tayade, K.N.; Wang, L.; Shang, S.; Dai, W.; Mishra, M.; Gao, S. Zirconium triflate grafted on SBA-15 as a highly efficient solid acid catalyst for ring opening of epoxides by amines and alcohols. Chin. J. Catal. 2017, 38, 758-766. [CrossRef]

12. Natongchai, W.; Khan, R.; Alsalme, A.; Shaikh, R. YCl3-Catalyzed Highly Selective Ring Opening of Epoxides by Amines at Room Temperature and under Solvent-Free Conditions. Catalysts 2017, 7, 340. [CrossRef]

13. Prathap, K.J.; Wu, Q.; Olsson, R.T.; Diner, P. Catalytic Reductions and Tandem Reactions of Nitro Compounds Using in Situ Prepared Nickel Boride Catalyst in Nanocellulose Solution. Org. Lett. 2017, 19, 4746-4749. [CrossRef] [PubMed]

14. Karimi, M.; Hajiashrafi, T.; Heydari, A.; Azhdari Tehrani, A. Terbium-organic framework as heterogeneous Lewis acid catalyst for $\beta$-aminoalcohol synthesis: Efficient, reusable and green catalytic method. Appl. Organomet. Chem. 2017, 31, e3866. [CrossRef]

15. Tan, N.; Yin, S.; Li, Y.; Qiu, R.; Meng, Z.; Song, X.; Luo, S.; Au, C.-T.; Wong, W.-Y. Synthesis and structure of an air-stable organobismuth triflate complex and its use as a high-efficiency catalyst for the ring opening of epoxides in aqueous media with aromatic amines. J. Organomet. Chem. 2011, 696, 1579-1583. [CrossRef]

16. Ollevier, T.; Lavie-Compin, G. An efficient method for the ring opening of epoxides with aromatic amines catalyzed by bismuth trichloride. Catal. Lett. 2002, 43, 7891-7893. [CrossRef]

17. Venkat Narsaiah, A.; Reddy, B.V.S.; Premalatha, K.; Reddy, S.S.; Yadav, J.S. Bismuth(III)-Catalyzed Hydrolysis of Epoxides and Aziridines: An Efficient Synthesis of vic-diols and $\beta$-Amino Alcohols. Catal. Lett. 2009, 131, 480-484. [CrossRef]

18. Bansal, S.; Kumar, Y.; Pippal, P.; Das, D.K.; Pramanik, P.; Singh, P.P. An efficient method for regioselective ring opening of epoxides by amines under microwave irradiation using $\mathrm{Bi}\left(\mathrm{NO}_{3}\right)_{3} \cdot 5 \mathrm{H}_{2} \mathrm{O}$ as a catalyst. New J. Chem. 2017, 41, 2668-2671. [CrossRef]

19. Torrelo, G.; Hanefeld, U.; Hollmann, F. Biocatalysis. Catal. Lett. 2014, 145, 309-345. [CrossRef]

20. Sheldon, R.A.; Brady, D. Broadening the Scope of Biocatalysis in Sustainable Organic Synthesis. ChemSusChem 2019, 12, 2859-2881. [CrossRef]

21. Woodley, J.M. New frontiers in biocatalysis for sustainable synthesis. Curr. Opin. Green Sustain. Chem. 2020, 21, 22-26. [CrossRef]

22. Sheldon, R.A.; Woodley, J.M. Role of Biocatalysis in Sustainable Chemistry. Chem. Rev. 2018, 118, 801-838. [CrossRef] [PubMed]

23. Sonawane, Y.A.; Phadtare, S.B.; Borse, B.N.; Jagtap, A.R.; Shankarling, G.S. Synthesis of diphenylamine-based novel fluorescent styryl colorants by Knoevenagel condensation using a conventional method, biocatalyst, and deep eutectic solvent. Org. Lett. 2010, 12, 1456-1459. [CrossRef] [PubMed]

24. Reetz, M.T. Lipases as practical biocatalysts. Curr. Opin. Chem. Biol. 2002, 6, 145-150. [CrossRef]

25. Dhake, K.P.; Qureshi, Z.S.; Singhal, R.S.; Bhanage, B.M. Candida antarctica lipase B-catalyzed synthesis of acetamides using [BMIm( $\left.\mathrm{PF}_{6}\right)$ ] as a reaction medium. Tetrahedron Lett. 2009, 50, 2811-2814. [CrossRef]

26. Jaeger, K.-E.; Eggert, T. Lipases for biotechnology. Curr. Opin. Biotechnol. 2002, 13, 390-397. [CrossRef]

27. Du, L.-H.; Long, R.-J.; Xue, M.; Chen, P.-F.; Yang, M.-J.; Luo, X.-P. Continuous-Flow Synthesis of $\beta$-Amino Acid Esters by Lipase-Catalyzed Michael Addition of Aromatic Amines. Catalysts 2020, 10, 432. [CrossRef]

28. Arsa, S.; Theerakulkait, C. Preparation, aroma characteristics and volatile compounds of flavorings from enzymatic hydrolyzed rice bran protein concentrate. J. Sci. Food Agric. 2018, 98, 4479-4487. [CrossRef]

29. Borude, V.S.; Shah, R.V.; Shukla, S.R. Synthesis of $\beta$-amino alcohol derivatives from phenols in presence of phase transfer catalyst and lipase biocatalyst. Curr. Chem. Lett. 2013, 2, 1-12. [CrossRef]

30. Gupta, P.; Bhatia, S.; Dhawan, A.; Balwani, S.; Sharma, S.; Brahma, R.; Singh, R.; Ghosh, B.; Parmar, V.S.; Prasad, A.K. Selective biocatalytic aminolysis of (+/-)-epichlorohydrin: Synthesis and ICAM-1 inhibitory activity of (S)-(+)-3-arylamino-1-chloropropan-2-ols. Bioorg. Med. Chem. 2011, 19, 2263-2268. [CrossRef]

31. Planchestainer, M.; Contente, M.L.; Cassidy, J.; Molinari, F.; Tamborini, L.; Paradisi, F. Continuous flow biocatalysis: Production and in-line purification of amines by immobilised transaminase from Halomonas elongata. Green Chem. 2017, 19, 372-375. [CrossRef]

32. Iemhoff, A.; Sherwood, J.; McElroy, C.R.; Hunt, A.J. Towards sustainable kinetic resolution, a combination of bio-catalysis, flow chemistry and bio-based solvents. Green Chem. 2018, 20, 136-140. [CrossRef] 
33. Valikhani, D.; Srivastava, P.L.; Allemann, R.K.; Wirth, T. Immobilised Enzymes for Sesquiterpene Synthesis in Batch and Flow Systems. Chem CatChem 2020, 12, 2194-2197. [CrossRef]

34. Britton, J.; Majumdar, S.; Weiss, G.A. Continuous flow biocatalysis. Chem. Soc. Rev. 2018, 47, 5891-5918. [CrossRef]

35. Van der Helm, M.P.; Bracco, P.; Busch, H.; Szymańska, K.; Jarzębski, A.B.; Hanefeld, U. Hydroxynitrile lyases covalently immobilized in continuous flow microreactors. Catal. Sci. Technol. 2019, 9, 1189-1200. [CrossRef]

36. Zhang, M.; Ettelaie, R.; Yan, T.; Zhang, S.; Cheng, F.; Binks, B.P.; Yang, H. Ionic Liquid Droplet Microreactor for Catalysis Reactions Not at Equilibrium. J. Am. Chem. Soc. 2017, 139, 17387-17396. [CrossRef] [PubMed]

37. Du, L.H.; Dong, Z.; Long, R.J.; Chen, P.F.; Xue, M.; Luo, X.P. The convenient Michael addition of imidazoles to acrylates catalyzed by Lipozyme TL IM from Thermomyces lanuginosus in a continuous flow microreactor. Org. Biomol. Chem. 2019, 17, 807-812. [CrossRef]

38. Fang, J.; Ke, M.; Huang, G.; Tao, Y.; Cheng, D.; Chen, F.-E. The Chapman rearrangement in a continuous-flow microreactor. RSC Adv. 2019, 9, 9270-9280. [CrossRef]

39. Arcus, V.L.; van der Kamp, M.W.; Pudney, C.R.; Mulholland, A.J. Enzyme evolution and the temperature dependence of enzyme catalysis. Curr. Opin. Struct. Biol. 2020, 65, 96-101. [CrossRef]

40. Wang, L.; Wang, Y.; Hu, C.; Cao, Q.; Yang, X.; Zhao, M. Preparation of Diacylglycerol-Enriched Oil from Free Fatty Acids Using Lecitase Ultra-Catalyzed Esterification. J. Am. Oil Chem. Soc. 2011, 88, 1557-1565. [CrossRef]

Publisher's Note: MDPI stays neutral with regard to jurisdictional claims in published maps and institutional affiliations. 